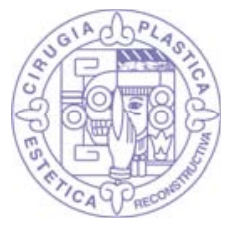

\title{
Mastectomía radical versus tumorectomía para el tratamiento de cáncer de mama: implicaciones bioéticas
}

\author{
Mastectomy versus lumpectomy in the treatment of breast cancer: \\ Bioethical implications \\ Dra. Roxanna Pérez Garmendia,* Dr. Juan Garzón Muvdi,* Dr. Isaac Baley Spindle,* \\ Dr. Eduardo Villaseñor Villalpando, * Dr. Cuauhtémoc Márquez Espriella*
}

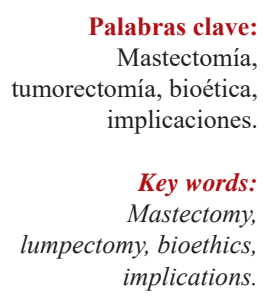

* Departamento de Cirugía Plástica y Reconstructiva. Hospital de Alta Especialidad PEMEX Sur.

Los autores de este artículo no tienen conflicto de intereses qué declarar.

Recibido:

12 abril 2018

Aceptado para publicar:

15 junio 2018

\section{RESUMEN}

Los avances científicos y biotecnológicos, así como la aparición de factores sociales y jurídicos en el mundo de la salud, han llevado, especialmente a la medicina, a reconsiderar su estudio y su práctica. Las expectativas actuales tanto de médicos como de los pacientes y de sus familiares sobrepasan los objetivos clásicos de la atención médica. Hasta hace unos años el tratamiento del carcinoma mamario era la mastectomía radical clásica, con o sin radioterapia postoperatoria. Sin embargo, en la actualidad con la tumorectomía, el criterio terapéutico se ha modificado, hay un mayor conocimiento de la historia natural de la enfermedad, existen fármacos más efectivos y en la medicina actual la importancia de la calidad de vida que tenga la paciente después del tratamiento es de suma importancia. Hay documentos que afirman que la mastectomía lleva consigo efectos indeseables a la mujer. La mastectomía, a diferencia de la tumorectomía, es un procedimiento quirúrgico más invasivo, es común encontrar en la paciente depresión, ansiedad, insomnio, vergüenza, inseguridad, ideas de suicidio y sentimientos de minusvalía, afectando de forma significativa su «calidad de vida». Se debe considerar todos los factores y las posibles consecuencias de los actos médicos y procurar no sólo tomar en cuenta la enfermedad como tal. Se necesita integrar para así poder brindar mejores resultados que se traducen en una mejor recuperación y calidad de vida.

\section{ABSTRACT}

Scientific and biotechnological advances, as well as the emergence of social and legal factors in the world of health, have led, especially medicine to reconsider its study and practice. The current expectations of doctors, patients and their families exceed the classic objectives of medical care. Until a few years ago, the treatment of mammary carcinoma was the classic radical mastectomy, with or without post-operative radiotherapy. However, at present with lumpectomy, the therapeutic criterion have been modified, there is a greater knowledge of the natural history of the disease, there are drugs that are more effective and in current medicine, the importance of the quality of life that a patient has after the treatment is of utmost importance. There are documents that claim that a mastectomy causes undesirable effects in women. Mastectomy, unlike lumpectomy, is a more invasive surgical procedure. It is common to find depression, anxiety, insomnia, shame, insecurity, suicidal thoughts and feelings of worthlessness in the patient, significantly affecting their "quality of life». All factors and the possible consequences of the surgeon's actions must be considered and efforts must be made not only to take into account the disease. It needs to be integrated in order to provide better results that translate into a better recovery and quality of life.

\section{INTRODUCCIÓN}

$\mathrm{L}$ os avances científicos y biotecnológicos, así como la aparición de factores sociales y jurídicos en el mundo de la salud, han llevado, especialmente a la medicina, a reconsiderar su estudio y su práctica. Las expectativas actuales tanto de médicos como de pacientes y sus familiares sobrepasan los objetivos clásicos de la atención médica. El modelo unilateral de decisión terapéutica ha tenido que cambiar por la relación entre las múltiples y diferentes áreas de la salud. La economía, política y derecho, entre otros, presionan las decisiones médicas, haciendo que las acciones de salud se vuelvan difíciles y en ocasiones polarizadas. 
Los objetivos tradicionales del acto médico están orientados básicamente a prevenir, conservar y restablecer la salud, evitar complicaciones y las secuelas $y$, especialmente, evitar la muerte. ${ }^{1}$ Los médicos han ido incorporando nuevos conocimientos y tecnologías a su práctica profesional y mediante habilidades y destrezas clínicas específicas los aplican, en general, en beneficio de los pacientes. ${ }^{2}$

Por este motivo, en las últimas décadas la relación médico-paciente, al igual que los fines propios del acto médico, se ha afectado, provocando en ocasiones que los pacientes al ser atendidos sufran una cierta deshumanización en su trato.

Paralelamente, la sociedad ha alcanzado (en muchos casos) un nuevo marco de valores: el respeto a la dignidad de la persona humana y la eliminación de desigualdades por credo, raza o posición social. Todo esto, junto con la libertad de conciencia y la gran pluralidad que ofrece el mundo actual, ha conducido al reconocimiento de los cuatro principios bioéticos enunciados por Beauchamp y Childress: ${ }^{3}$ no maleficencia, justicia, beneficencia, así como el derecho a participar en las decisiones que a cada uno conciernen (autonomía), entre otros principios como el de proporcionalidad terapéutica y de totalidad corporal, que afecten en forma determinante el cauce a seguir para el tratamiento de las enfermedades.

Unos de los principales problemas de salud pública es sin duda el cáncer mamario. Es el más frecuente en mujeres de países desarrollados como de los en desarrollo. La incidencia de cáncer de mama está aumentando en el mundo en desarrollo debido a la mayor esperanza de vida y al aumento de la urbanización, entre otros, y representa el $16 \%$ de todos los cánceres femeninos. Se estima que en 2004 murieron 519,000 mujeres por cáncer de mama y, aunque se considera como una enfermedad del mundo desarrollado, la mayoría de las defunciones por esa causa (69\%) se registra en los países en desarrollo (OMS: Carga Mundial de Morbilidad 2004).

Las tasas de supervivencia del cáncer mamario varían mucho en todo el mundo, desde el $80 \%$ o más en América del Norte, Suecia y Japón, pasando por un $60 \%$ aproximadamente en los países de ingresos medios, hasta cifras inferiores al $40 \%$ en los países de ingresos bajos. ${ }^{4}$

Las bajas tasas de supervivencia observadas en los países poco desarrollados se pueden explicar principalmente por la falta de programas de detección precoz, que hace que un alto porcentaje de mujeres acudan al médico con la enfermedad muy avanzada, pero también por la falta de servicios adecuados de diagnóstico y tratamiento. ${ }^{5}$

Hasta hace unos años el tratamiento del carcinoma mamario era la mastectomía radical clásica, con o sin radioterapia postoperatoria. Sin embargo, en la actualidad el criterio terapéutico se ha modificado, hay un mayor conocimiento de la historia natural de la enfermedad, existen fármacos más efectivos y en la medicina actual la importancia de la calidad de vida que tenga la paciente después del tratamiento juega un papel primordial.

Se ha comprobado que este tipo de cirugía no tiene ventajas significativas sobre la tumorectomía. En estudios observacionales realizados en mujeres con diagnóstico de cáncer de mama, se concluyó que la mastectomía no tenía ventaja pronóstica sobre la tumorectomía acompañada de radiación. Se estudió a 112,000 mujeres diagnosticadas con la enfermedad entre los años 1994 y 2004 y se monitorearon las secuencias de la mastectomía radical versus tumorectomía. El 55\% de las mujeres estudiadas tuvo tratamiento conservador (tumorectomía con radiación) y el $45 \%$ restante fue sometido a tratamiento radical (mastectomía). ${ }^{6}$ Después de un seguimiento no menor de nueve años, se concluyó que el pronóstico y la sobrevida es igual entre ambos tratamientos; ${ }^{7}$ sin embargo, la mastectomía tiene complicaciones psicológicas, personales y en ocasiones médicas más severas que las que tienen las pacientes con tratamiento conservador. Estas conclusiones no difieren de las establecidas en 1990 por los Institutos Nacionales de Salud de los Estados Unidos $(\mathrm{NIH}$, por sus siglas en inglés).

Existen documentos que afirman que la mastectomía tiene consigo efectos indeseables para la mujer. La mastectomía, a diferencia de la tumorectomía, es un procedimiento quirúrgico más invasivo; es común encontrar en la paciente depresión, ansiedad, insomnio, 
vergüenza, inseguridad, ideas de suicidio y sentimientos de minusvalía, ${ }^{8}$ que afectan en forma significativa su «calidad de vida».

La calidad de vida es un término utilizado ampliamente por sociólogos, filósofos, políticos y profesionales de la salud. Se empleó por primera vez en la Ética a Nicómaco, escrita por Aristóteles en el año 330 a.C. En ella, se reconoce la relación directa entre felicidad y calidad de vida. ${ }^{9}$

Al considerar esto, se entiende que dicho concepto se refiere a la disfunción física y emocional del individuo, así como los aspectos subjetivos como la satisfacción, que de la misma manera se ve afectada en forma indirecta por la presencia de incapacidades o pérdida de habilidades. ${ }^{10}$ Todos estos rubros se consideran y agrupan para formar la sensación de bienestar del paciente y no la opción del proveedor sanitario.

Toda secuela postquirúrgica, sea física o psicológica, repercute considerablemente en la adaptación de la paciente y en su calidad de vida. Se debe considerar todos los factores y las posibles consecuencias de los actos médicos y procurar no tomar sólo en cuenta la enfermedad como tal. Se necesita integrar para poder brindar mejores resultados que se traduzcan en una mejor recuperación y por tanto «calidad de vida».

La imagen corporal es un concepto que se ha interpretado en muchas formas; sin embargo, la definición más aceptada es la dada por Prince, ${ }^{11}$ quien la describe como «la totalidad de cómo se siente uno y piensa en su propio cuerpo y apariencia». Esta definición incorpora tres elementos de gran importancia:

1. La realidad del cuerpo, que es uno de los puntos más objetivos donde se puntualizan los cambios anatómicos, como en el caso de las amputaciones.

2. El cuerpo ideal, que tiene un carácter subjetivo y depende de cada persona.

3. La presentación del cuerpo, que se refiere a cómo se presenta el cuerpo en el ambiente externo considerando los conceptos de belleza dictados por la sociedad.

Podemos afirmar, por tanto, que la mastectomía afecta superlativamente la calidad de vida de los pacientes. ${ }^{12}$ Sin embargo, se ha argumentado que la cirugía reconstructiva de la mama mejora la imagen y apariencia física, pero el estado de ánimo, la ansiedad e incertidumbre y el sentimiento de minusvalía no mejoran significativamente después de la reconstrucción mamaria. ${ }^{13,14}$

Otros efectos negativos que se presentan en pacientes sometidas a mastectomía radical son los que se asocian con su sexualidad. Los efectos secundarios más comunes de esta surgen del daño a los sentimientos sobre el atractivo de la mujer. En nuestra cultura se nos enseña a ver las mamas como una parte básica de la belleza y femineidad. Si se extirpa una mama es posible que una mujer se sienta insegura, que se cuestione si su pareja la va aceptar y a encontrar sexualmente atractiva. Otro de los efectos secundarios resultantes de esta mutilación es la pérdida de sensibilidad, así como dolor crónico del tórax y hombros. Es mucho el dolor físico y emocional por el que tienen que pasar las mujeres que fueron sometidas a este procedimiento quirúrgico radical.

La cirugía conservadora de la mama acompañada de radiación se percibe por la comunidad científica como un tratamiento superior para atender el cáncer de mama de diagnóstico temprano, ya que es menos invasivo, preserva la mama y, por ende, reduce alteraciones sexuales, problemas cosméticos y secuelas psicológicas postquirúrgicas. ${ }^{15}$ Existe evidencia empírica que sugiere que las pacientes sometidas a terapias que conservan la mama se adaptan con más facilidad y regresan a su vida cotidiana más temprano. ${ }^{16}$

Por lo anterior, es preciso que ante un diagnóstico de cáncer de mama se estudien todas las posibilidades existentes en forma razonada y fundamentada, apoyándose al mismo tiempo en la experiencia, para el tratamiento del padecimiento y al mismo tiempo analizar los efectos secundarios físicos y emocionales que tendrán las pacientes. ${ }^{17}$

En la ética médica, el principio de no maleficencia (primum non nocere) es el más importante; este principio dicta que el médico se debe abstener de ejercer acciones que puedan dañar o perjudicar a sus pacientes, sea de manera directa o indirecta. Este no es un principio exclusivo del área médica, pero con ella 
adquiere una interpretación especial, ya que en muchas ocasiones los procedimientos médicos se tienen que regir por el principio de doble efecto (hacer un daño para hacer un bien); es decir, el principio por el que es posible realizar un acto que produzca un mal resultado. ${ }^{18}$

En el caso del tratamiento de cáncer de mama por medio de la mastectomía radical, el efecto malo sería la extirpación total de la mama, pero con el objetivo claro de salvar la vida y tener un pronóstico de vida adecuado y buena calidad de vida para la paciente. Sin embargo, en caso de que existiera otro medio para lograr los mismos fines, como en la tumorectomía y se tomara la decisión argumentando la seguridad y las posibilidades no fundamentas de un mayor éxito en la recuperación del paciente, este principio se vería afectado al igual que el principio de no maleficencia.

Por otro lado, el principio de no maleficencia se vincula fuertemente con el principio de beneficencia dictando que prevalezca el beneficio sobre el perjuicio.

Ante este dilema, tendría que preguntarse si el médico está promoviendo el mejor interés del paciente.

Cuando se realiza un diagnóstico de cáncer, lo primero que llega a la mente de todo paciente es removerlo y ese pensamiento es lógico y entendible. El cáncer de mama diagnosticado tempranamente no es la excepción.

Cuando existe más de una opción terapéutica, como en este caso, se debe procurar considerar la decisión del paciente e informarle de todos los aspectos de ambos tratamientos. El médico posee una formación que se acompaña de conocimientos que no tiene el paciente, por lo que sabe y decide lo que es conveniente para éste.

Es necesario que el médico informe al paciente con claridad, completa y concisamente todos los aspectos a considerar ante los diversos procedimientos, tratamientos y pronósticos, así como los efectos secundarios de cada uno de ellos, para que el paciente pueda tomar la decisión que vea más adecuada para él y de esta manera respetar su autonomía y libertad.

De esta manera, el modelo para la toma de decisiones deja de ser un modelo paternalista, donde el médico decide cuál es el paso a seguir y pasa a ser un modelo de carácter informativo.
En este modelo informativo el profesional de la salud debe externar a su paciente toda la información que involucre ambos procedimientos y permitir que el paciente y sus familiares tomen la decisión que les parezca conveniente. Gadagnoli y Ward concluyen que la participación de los pacientes en la toma de decisiones se justifica en grados variables y que los médicos tienen el deber de invitar a que tomen decisiones, siempre y cuando exista más de una opción terapéutica efectiva, como en el caso de cáncer de mama. ${ }^{19}$

Si no se le brinda al paciente información concisa y se le explican detalladamente las alternativas terapéuticas para su padecimiento, como en el cáncer de mama, estamos violando estos principios de los que el paciente tiene derecho. Sólo se está tratando la enfermedad y no el padecimiento; estamos dejando a un lado todos los factores que se asocian al cáncer y al tratamiento radical. La preocupación radica en que si existen dos alternativas terapéuticas con pronósticos de sobrevida similares, siga en aumento el uso del procedimiento invasivo, sin presentar evidencia científica que avale dicha tendencia.

Si se deja de considerar el bienestar integral de todo individuo que tiene enfermedades como el expuesto en este caso, entonces estamos tratando enfermedades y dejamos de atender personas. Sólo se administra un tratamiento específico para la enfermedad y no se emite una terapéutica integral, considerando todos los factores que afectan al individuo.

La cirugía de mama es un tema complejo desde el punto de vista clínico, de investigación y ético. Tenemos que aceptar que el paciente es autónomo y que dentro de sus derechos cuenta con la percepción y explicación detallada de su padecimiento y las opciones terapéuticas médicas o quirúrgicas de las que dispone, así como sus pros y contras. Esto es básicamente proporcionarles las herramientas necesarias para la toma de decisiones.

Con el advenimiento de nuevas técnicas quirúrgicas y la disposición de mejores y más sofisticados equipos médicos, como en la cirugía conservadora y reconstructiva de la mama, ha iniciado una tendencia de brindar atención holística e integral. Los pacientes empiezan a informarse cada vez más y a considerar la 
cirugía menos invasiva. Se deja a un lado la costumbre de sólo evaluar los índices de mortalidad y pronóstico y se les suman los índices se satisfacción de calidad de vida.

Donabedian argumentó hace más de 30 años que para poder evaluar la calidad del servicio de salud se tenía que incluir no sólo la capacidad de preservar la vida y la salud, sino la calidad de vida postratamiento y la satisfacción del paciente. ${ }^{9}$

En otras palabras, los servicios sanitarios no se pueden considerar de calidad a menos que el paciente esté satisfecho. Hoy, con el incremento de la competitividad en el sector salud, el tema de calidad del servicio es directamente proporcional al resultado en la calidad de vida del paciente. Este argumento vuelve el tema en algo muy controvertido entre los profesionales de la salud y entre los pacientes. Todavía tenemos que recorrer un largo camino para mejorar la calidad en la atención que se brinda a las mujeres con cáncer de mama. Se tiene que explicar a detalle al paciente todas las modalidades terapéuticas disponibles y dejar en el pasado la falta de información en el que se tiene a los pacientes.

Es necesario informar de forma adecuada la igualdad en las posibilidades de sobrevida a las pacientes con cáncer de mama, con la mastectomía radical y la tumorectomía asociada a radioterapia. Considerando esto, los esfuerzos para una buena praxis médica se deben concentrar principalmente en brindar una buena calidad de vida a las pacientes con diagnóstico temprano de cáncer de mama. Del mismo modo, se debe considerar brindar una evaluación psicológica adecuada en el preoperatorio y brindar apoyo psicológico adecuado después de la crisis.

Está en las manos del personal de salud ver más allá del frío tratamiento quirúrgico contra el cáncer de mama e implementar una atención holística integral.

Con toda la evidencia presentada se ha argumentado que la cirugía reconstructiva de la mama mejora la imagen y la apariencia física, pero el estado de ánimo, ansiedad, incertidumbre, el sentimiento de minusvalía y en general la calidad de vida no mejoran de manera significativa después de la reconstrucción mamaria. ${ }^{13,14}$

\section{REFERENCIAS}

1. Casas M. Bases bioéticas. México: Trillas; 2008.

2. Goic GA. Tendencias de la medicina actual. Rev Med Chile 1999; 127 (9): 1136-1138.

3. Beauchamp TL, Childress JF. Principles of biomedical ethics: oxford university press. 1979, p. 191.

4. Coleman MP, Quaresma M, Berrino F, Lutz JM, De Angelis R, Capocaccia R, Baili P, Rachet B, Gatta G, Hakulinen T, Micheli A, Sant M et al. Cancer survival in five continents: a worldwide population-based study (CONCORD). Lancet Oncol 2008; 9 (8): 730-756.

5. http://www.who.int/topics/cancer/breastcancer/es/

6. Hwang ES, Lichtensztajn DY, Gomez SL, Fowble B, Clarke CA. Survival after lumpectomy and mastectomy for early stage invasive breast cancer: the effect of age and hormone receptor status. Cancer 2013; 119 (7): 1402-1411.

7. National Institutes of Health Consensus Development Conference Statement: The treatment of Early Stage Breast cancer. Bethesda, MD: Author; 1990.

8. Wording JW, Weisman AD. The fallacy in post mastectomy depression. Am J Med Sci 1977; 273: 169-175.

9. Schipper H, Clinch J, Olweny C. Quality of life and pharmacoeconomics in clinical trials. Quality of life studies: definitions and conceptual issues. Lippincott: Philadelphia 2aㅡ ed. 1996, pp. 25-35.

10. Patrick D, Erickson P. Health status and health policy; Quality of life in health care evaluation and resource allocation. Oxford University Press. New York, 1993.

11. Price B. A model for body-image care. J Adv Nurs 1990; 15: 585-593.

12. Rytina S. Imagined ugliness. Sci Am Mind 2008; 19: 72-77.

13. Hart S, Meyerowits BE, Apolone G, Masconi P, Liberati A. Quality of life among mastectomy patients using external breast prostheses. Tumori 1997; 83: 581-586.

14. Wellish DK, Dimatteo R, Silversteim M. Psychosocial outcomes of breast cancer therapies: lumpectomy versus mastectomy. Psychosomatics 1989; 30: 365-373.

15. Margolis GJ, Goodman RL, Rubin A, Pajac TF. Psychological factors in the choice of treatment breast cancer. Psychosomatics 1989; 30 (2): 192-197.

16. Levy SM, Herberman RB, Lee JK Lippman ME, D Angelo T. Breast conservation versus mastectomy: distress sequel as a function of choice. J Clin Oncol 1989; 7 (3): 367-375.

17. González F. Tratamiento quirúrgico del carcinoma mamario. Rev Inst Nac Cancer Méx 1986; 32 (3/4): 212-217.

18. http://www.bioeticahoy.com.es/2010/02/principio-dedoble-efecto.html

19. Guadagnoli E, Ward P. Patient participation in decisionmaking. Soc Sci Med 1998; 47 (3): 329-339.

\author{
Correspondencia: \\ Dr. Juan Garzón Muvdi \\ Blvd. Adolfo Ruiz Cortines Núm. 4091, \\ Col. Fuentes del Pedregal, \\ Alcaldía Tlalpan, 14140, \\ México, Ciudad de México \\ Tel: 8441004083. \\ E-mail: garzonmuvdi@gmail.com
}

